whether it is ever possible for the first and second fundamental forms of a surface to be the second and first respectively of a second surface. In answer it is found that there is a class of surfaces possessing this property ; that they are ruled surfaces with imaginary generatrices; that the sphere of radius unity is the only real surface of the class; and, furthermore, that the second surface differs from the first only by a translation in space.

Columbia University.

F. N. Cole.

\title{
GREEN'S FUNCTIONS IN SPACE OF ONE DIMENSION.
}

BY PROFESSOR MAXIME BÔCHER.

(Read before the American Mathematical Society, February 23, 1901.)

I WISH to make a brief communication to the Society of some results which I have obtained, reserving proofs and further developments for another occasion.

By a Green's function is ordinarily understood a solution of Laplace's equation which vanishes on the boundary of a certain region, and within this region is discontinuous at only one point, where it becomes infinite like $1 / r$ or $\log r$, according as we are dealing with Laplace's equation in three or in two dimensions. We may speak of this as a Green's function of the first kind, in distinction to the generalized Green's function for which more complicated conditions than the mere vanishing of the function are imposed on the boundary of the region.

This fundamental conception has been generalized by replacing Laplace's equation on the one hand by other homogeneous linear partial differential equations of the second order (cf. Encyklopädie, volume 2, p. 516); on the other hand by the ordinary differential equation $d^{2} y / d x^{2}=0$, which may be called Laplace's equation in space of one dimension (cf. Burkhardt, Bulletin de la Société mathématique de France, volume 22 (1894), p. 71). This suggests at once the possibility of considering in place of Laplace's equation the general ordinary homogeneous linear differential equation of the second order. I find that we can not only do this, 
but can go further by considering at once linear differential equations of the $n$th order

$$
\frac{d^{n} y}{d x^{n}}+p_{1} \frac{d^{n-1} y}{d x^{n-1}}+\cdots+p_{n} y=0 \quad(n \geqq 2) .
$$

We will assume here, in order to avoid all complications, that $p_{1}, p_{2}, \cdots, p_{n}$ are real functions of the real variable $x$ which are continuous at every point of the interval

$$
a \leqq x \leqq b \text {. }
$$

Let $\xi$ be a point between $a$ and $b$, and let $k$ be a positive integer less than $n(1 \leqq k \leqq n-1)$.

We seek now a function $G(x)$ having the following properties :

(a) At every point of $(J)$, except $\xi$, the function $G$ is continuous, has continuous derivatives of the first $n$ orders, and satisfies (1).

(b) At the point $a, G$ and its first $n-1$ derivatives satisfy $k$ linearly independent, but otherwise arbitrarily chosen, homogeneous linear relations.

(c) At the point $b, G$ and its first $n-1$ derivatives satisfy $n-k$ linearly independent, but otherwise arbitrarily chosen, homogeneous linear relations.

(d) At $\xi, G$ and its first $n-2$ derivatives are continuous.

(e) At $\xi, G$ has a derivative $D_{+}$of order $n-1$ taken in the positive direction, and a derivative $D_{-}$of order $n-1$ taken in the negative direction, and

$$
D_{+}-D_{-}=-1 \text {. }
$$

It turns out that in general there exists one and only one function satisfying these conditions. This function we call a Green's function, and denote it by $G(x, \xi)$. The effect of interchanging $x$ and $\xi$ is to change (1) into the adjoint equation (cf. Schlesinger's Handbuch, Vol. I., p. 53) and in general to alter the relations $(b)$ and $(c)$; otherwise conditions $(a)-(e)$ remain true.

I will mention here only one application of the Green's function thus defined :

The solution of the non-homogeneous equation

$$
\frac{d^{n} y}{d x^{n}}+p_{1} \frac{d^{n-1} y}{d x^{n-1}}+\cdots+p_{n} y=p,
$$

which satisfies at $a$ and $b$ the same conditions which are satisfied there by $G$, is given by the formula 


$$
y=-\int_{a}^{b} G(x, \xi) p(\xi) d \xi .
$$

A special case deserves mention on account of its simplicity, viz., the case where conditions $(b)$ reduced to

$$
G(a, \xi)=G^{\prime}(a, \xi)=\cdots=G^{[k-1]}(a, \xi)=0
$$

and conditions $(c)$ to

$$
G(b, \xi)=G^{\prime}(b, \xi)=\cdots=G^{[n-k-1]}(b, \xi)=0,
$$

accents denoting differentiation with regard to $x$. One of the simplifications introduced by this specialization is that when $x$ and $\xi$ are now interchanged, the effect is to interchange $a$ and $b$ in conditions $(b)$ and $(c)$. It will be seen that for a given equation (1) and a given interval $(J)$ there are $n-1$ Green's functions of this special sort, obtained by giving to $k$ the values $1,2, \cdots, n-1$. These functions might properly be called Green's functions of the first kind, since when $n=2$ there is only one of them, namely the Green's function, which vanishes at both ends of $(J)$.

Göttingen, Germany, February 8, 1901.

\section{ON A SYSTEM OF PLANE CURVES HAVING FACTORABLE PARALLELS.}

BY DR. VIRGIL SNYDER.

(Read before the American Mathematical Society, December 28, 1900.)

The type of scrolls contained in a linear congruence, and having factorable asymptotic lines, gives rise to a class of plane curves whose parallels have a similar property.

It has been shown * that the spherical images of such scrolls have factorable lines of curvature and a method was given by means of which a scroll of this type could be transformed into an annular surface having only plane lines of curvature, the curve of each section breaking up into two factors. The planes of the system all belong to the same axial pencil, and the locus of centers of the generating spheres lies in the bisecting plane of the angle between the two plane directors.

*V. Snyder, "On a special form of annular surface." Amer. Jour. of Math., vol. 23 . 